\title{
Otoplasty Morbidity
}

\author{
Katerina Anesti ${ }^{*}$, A. L. H. Moss \\ Guy's and St. Thomas' NHS Trust, London, UK. \\ Email: "katanest@gmail.com
}

Received May $7^{\text {th }}, 2012$; revised June $10^{\text {th }}, 2012$; accepted July $15^{\text {th }}, 2012$

\begin{abstract}
Background: Auricular deformities, specifically prominent ears are relatively frequent. Although the physiologic consequences are negligible, the aesthetic and psychological impact on a child's self-image can be substantial. The purpose of our study was to examine the post-operative morbidity of otoplasty, analyse the revision rate and identify, if possible, a gold standard procedure. Methods: Retrospective analysis of the results of 104 operations for correction of prominent ears in 24 months that were performed in one NHS Hospital in London, UK. Complications were recorded and analysed. Cases requiring revision were reviewed further, according to technique, seniority of Surgeon and whether a trainee was supervised or not. Results: Of 104 patients, 57 were male and 47 were female. Age ranged from 4 to 60 years. Peak incidence for the primary operation was identified in the early adolescence for both sexes. Total skeletonisation of the cartilage was used in 26 patients (25\%). The anterior scoring technique was used in 76 patients (73\%). Cartilage holding sutures were used in 52 patients (50\%). Complications were recorded in 32 patients, while 11 patients had more than one complications. There was no significant difference in the complication rate between the most popular methods. (Anterior scoring with or without holding sutures, not including Mustardé type, versus total cartilage skeletonisation technique). Conclusion: The multitude of different approaches indicates that there is not clearly definitive technique for correcting prominent ears. It is preferable that the surgeon is comfortable with multiple techniques (to tailor the correction to each individual patient and deformity).
\end{abstract}

Keywords: Otoplasty; Revision Rate; Complication Rate; Otoplasty Fascia Flap

\section{Introduction}

Prominent ears are the most common congenital deformity in the head and neck region, with an incidence of about five per cent in white population [1]. It is inherited as an autosomal dominant trait and is commonly caused by a combination of two defects:

1) Underdevelopment of antihelical folding.

2) Overdevelopment of the conchal wall [2].

Strong feelings about this deformity are culture bound and this might explain why in the Far East, where it often means a sign of good fortune, its correction is an unpopular procedure, while in this part of the world prominent ears often seem to be considered a sign of idiocy [3].

More than 150 operative techniques have been described in the past 100 years for correction of protruding ears, all claiming to be excellent and giving excellent results [2].

Otoplasty is routinely performed as an elective cosmetic procedure but it seems that there is no single widely accepted procedure that has been adopted by most surgeons. There are a number of refinements in the sur-

${ }^{*}$ Corresponding author. gical technique for prominent ears that have arisen out of the desire to improve cosmetic results and reduce the complication rate. They can be grouped into operations that involve:

1) Excision of cartilage.

2) Molding the ear with sutures (Mustardé).

3) Molding the ear with scoring or sculpting of cartilage (Stenstrom).

4) Combination of any of the above.

5) Nonsurgical-molding with splint (Gault).

The aim of our study was:

1) To examine the post-operative morbidity of otoplasty.

2) To analyse the revision rate and compare with the literature.

3) To investigate the complication rate in relation to the seniority of the surgeon and the technique.

4) To identify, if possible, a gold standard procedure.

\section{Patients and Methods}

Data were gathered retrospectively from case notes, day surgery proformas, dressing clinic notes and outpatient clinics, to identify post-operative morbidity. The age and 
sex of the patients, type of anaesthetic, grade of Surgeon (whether supervised or not) and technique used were noted. Specific parameters were assessed, including postoperative bleeding, haematoma, infection, skin necrosis, wound dehiscence, scarring, dysaesthesia and need for revision surgery (under or over correction, secondary deformities, asymmetry). A total of 104 consecutive operations for correction of prominent ears were performed in one hospital over a 24 months period.

\section{Results}

Of 104 patients, 57 were male and 47 were female. Distribution of operations according to Seniority of Surgeon is demonstrated on Figure 1. Ages ranged from 4 to 60 years. Peak incidence for the primary operation was identified in the early adolescence for both sexes (Figure 2). All patients were placed into a head bandage for 7 10 days and they attended for wound check and removal of sutures in the Dressing Clinic. They were advised to use a light head bandage support (woollen hat) usually for 1 month at night. The follow-up ranged between 6 weeks to 6 months. 15 patients $(14.4 \%)$ were lost to follow up.

Bilateral prominent ear correction was performed on 96 patients and unilateral on 8. A total of 98 had their operation under general anaesthesia, and 6 under local. All the surgeons used local anaesthetic, usually with Adrenaline, for infiltration and hydro-dissection. The initial approach was common, through a posterior dump bell skin incision. Total skeletonisation of the cartilage, as it was described by Stenstrom and Heftner [4], was used in 26 patients (25\%). The skin is undermined from the posterior incision, around the entire cartilage framework, without any disruption between the helical rim and the antihelix. The anterior scoring technique, as it was proposed by Chongchet [5], which is the most popular technique in UK, was used in 76 patients (73\%). It is a form of cartilage cutting technique that uses a cartilage incision between the helical rim and the antihelix to gain access to the anterior surface of the auricular cartilage. Cartilage holding sutures were used in 52 patients (50\%), (Figure 3).

Complications were recorded in 32 patients, (Table 1). Haemorrhage noted in 4 patients (3.9\%) and 3 of them had to return to theatre for drainage of haematoma. Skin necrosis was observed in 5 patients (4.8\%), all of whom required repeated outpatient dressings with satisfactory healing. No keloid or hypertrophic scarring was recorded. Residual deformity was noted in 14 patients (13.4\%) and was mainly due to prominence of the upper third of the ear. In 6 (5.8\%) of them anterior scoring with holding sutures had been used as the initial procedure. A total of 6 patients (5.8\%) have had or awaiting revision surgery to improve the cosmetic result. Equal distribution of patients between the different techniques was noticed for the revision rate. Also for the Seniority of the Surgeon that performed the primary procedure.

The Junior Trainees seemed to adopt the favourable technique of their Consultant, while more Senior Trainees used the technique of their personal preference, (Table 2 and Figures 4 and 5 demonstrate the complication rate according to method and Surgeon).

\section{Total Operations by Surgeon}

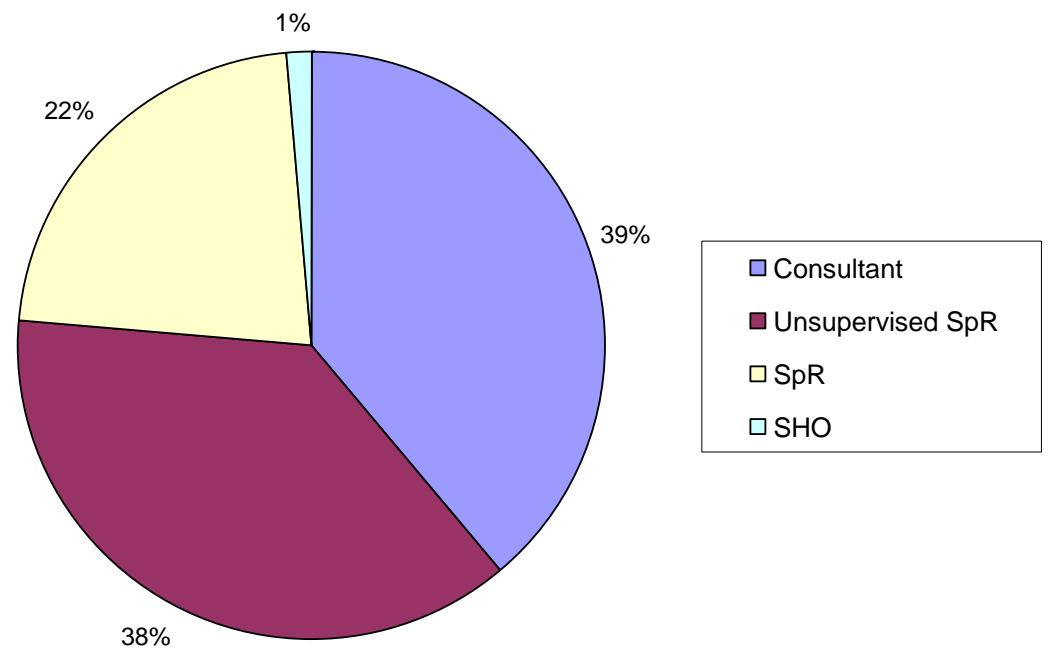

Figure 1. Total number of procedures by seniority of surgeon: consultant (C), unsupervised registrar (UnSpR), supervised registrar (SpR), senior house officer (SHO). 
Distribution by Age and Gender

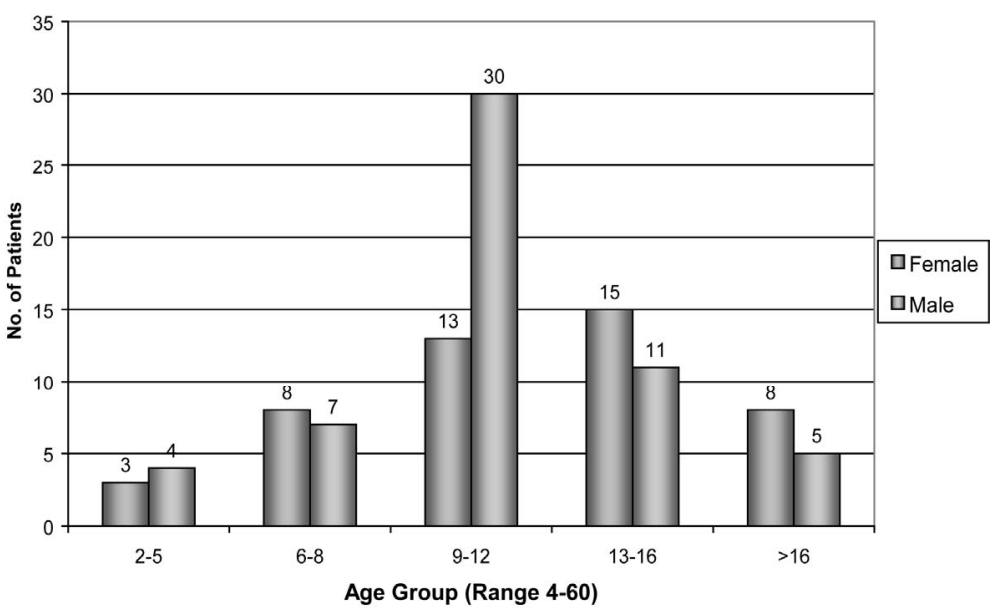

Figure 2. Distribution of procedures by age and gender.

Total Method by Surgeon

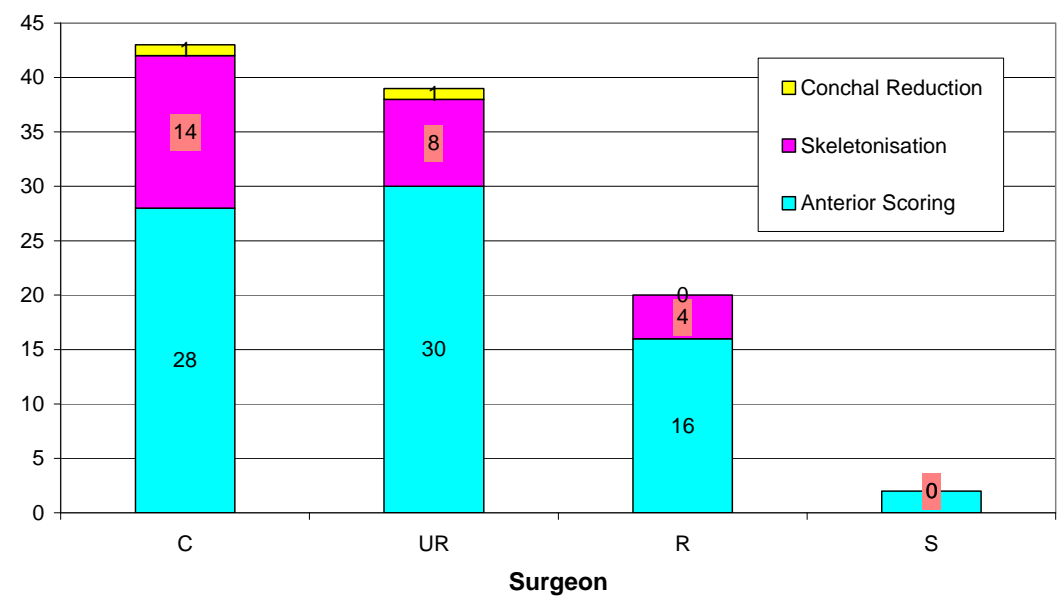

Figure 3. Otoplasty method by level of seniority of surgeon.

Complications by Method (\%)

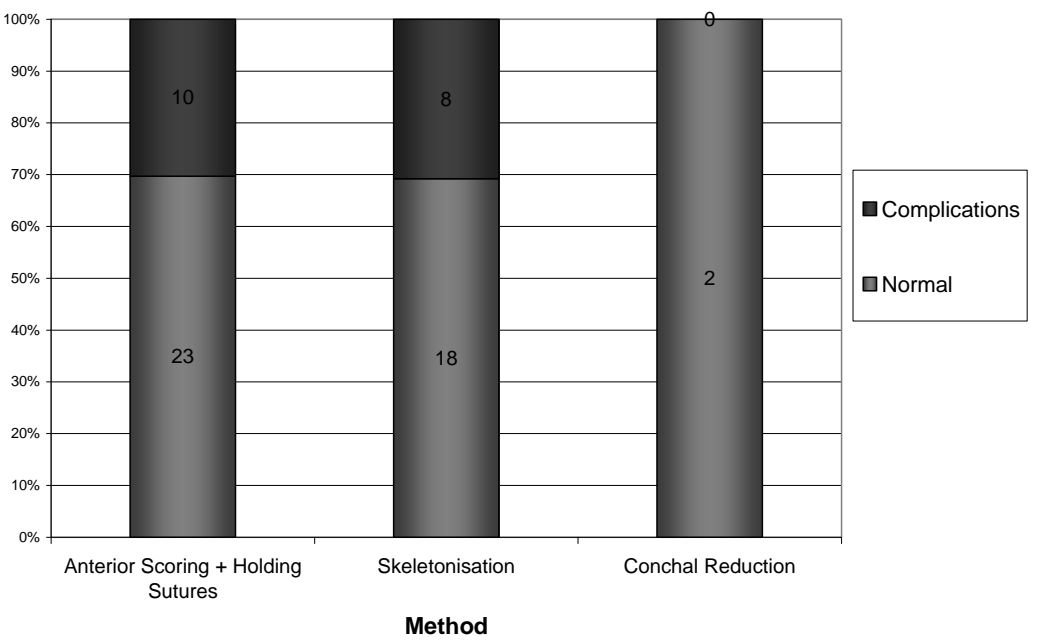

Figure 4. Complications by otoplasty method. 


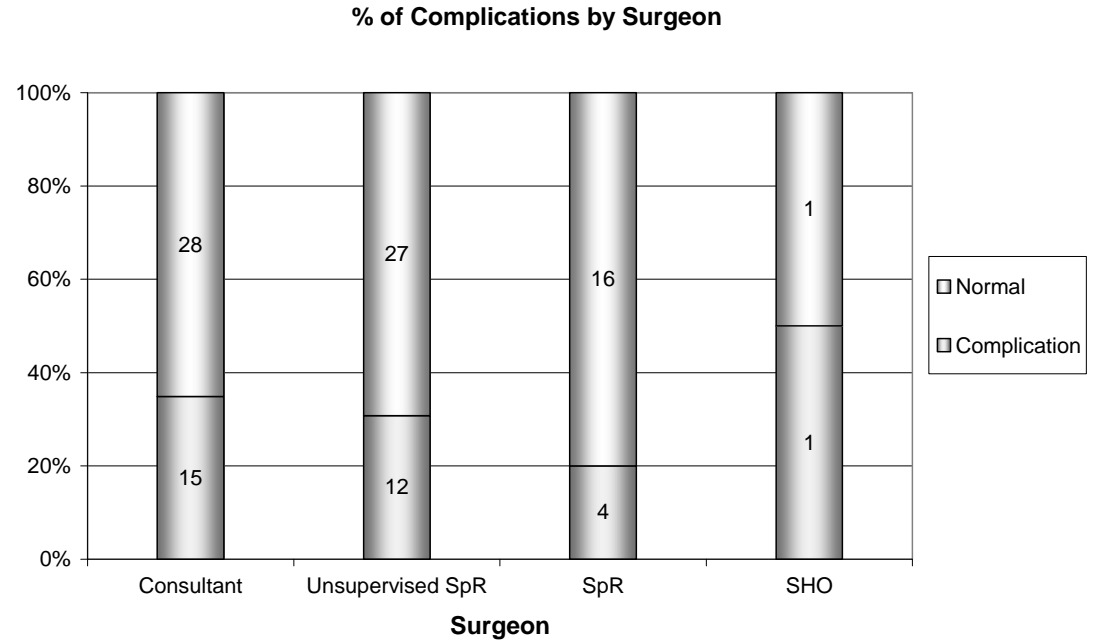

Figure 5. Complications by seniority of surgeon.

Table 1. List of complications.

\begin{tabular}{|c|c|c|}
\hline \multicolumn{3}{|c|}{ Complications Table } \\
\hline Type & Ear/Pole & $\begin{array}{c}\text { Occurrence } / \% \text { of } \\
\text { total }\end{array}$ \\
\hline \multirow[t]{2}{*}{ Bleeding/Haematoma } & Left & $1(1.0 \%)$ \\
\hline & Right & $3(2.9 \%)$ \\
\hline Infection & & $3(2.9 \%)$ \\
\hline Skin necrosis & & $5(4.8 \%)$ \\
\hline Keloid & & $0(0 \%)$ \\
\hline Hypertrophic scarring & & $0(0 \%)$ \\
\hline Pain & & $4(3.8 \%)$ \\
\hline Dysaesthesia & & $4(3.8 \%)$ \\
\hline \multirow[t]{3}{*}{ Deformity recurrence } & Right & 7 (6.7\%) \\
\hline & Left & $5(4.8 \%)$ \\
\hline & Both & $1(1.0 \%)$ \\
\hline Cartilage irregularity & & $3(2.9 \%)$ \\
\hline Severe asymmetry & & $1(1.0 \%)$ \\
\hline Wound dehiscence & & $2(1.9 \%)$ \\
\hline Abscess & & $2(1.9 \%)$ \\
\hline Other & & $3(2.9 \%)$ \\
\hline Re-operation & & $5(4.8 \%)$ \\
\hline Revision & & $6(5.8 \%)$ \\
\hline
\end{tabular}

Other complications: Wound dehiscence in 2 patients that was treated conservatively. (Both patients had skin closure with Prolene subcuticular sutures that were removed in 7 days).

Abscess formation in 2 cases. (In both occasions in-
Table 2. Complications by level of surgeon (C: consultant; SpR: supervised registrar; UnSpR: unsupervised registrar; SHO: senior house officer).

\begin{tabular}{ccccccc}
\hline Method & C & SpR & UnSpR & SHO & Total & $\begin{array}{c}\text { No. of } \\
\text { complications }\end{array}$ \\
\hline Anterior scoring & 13 & 7 & 21 & 2 & 43 & 14 \\
$\begin{array}{c}\text { Anterior scoring + } \\
\text { Holding sutures }\end{array}$ & 15 & 9 & 9 & 33 & 10 \\
$\begin{array}{c}\text { Total skeletonisation } \\
\text { Conchal excision }\end{array}$ & 14 & 4 & 8 & 26 & 8 \\
$\begin{array}{c}\text { Total No. of } \\
\text { complications }\end{array}$ & & & & & & 32 \\
\hline
\end{tabular}

fection that required reoperation had been sustained postoperatively). In one occasion the infection was due to infected haematoma.

Cartilage irregularity noticed in 3 occasions not requiring secondary procedure, and 4 patients complained of persistent pain, (at least for 4 months postoperatively).

\section{Discussion}

The goals of otoplasty have been well described by McDowell: [6].

- Complete correction of upper third protrusion.

- Visibility of the helix rim beyond the antihelix when viewed from the front.

- A smooth and regular helix.

- Prevention of distortion or decrease in the depth of postauricular sulcus.

- Placement of the ear at the correct distance from the head and not overly close.

- Bilateral symmetry-the difference in the helix-tomastoid distance between sides should be no greater than $3 \mathrm{~mm}$. 
Many authors have published reports in the incidence of complications after otoplasty. Our chart review has the same limitations as others for the length of the follow up. The incidence of early complications in our study has been compared with other published series. The incidence of postoperative bleeding (3.9\%) is similar for most series. On further study, the routine practice of giving a non steroid ant inflammatory post-operatively (known for postoperative bleeding) has been stopped until 8 hrs following surgery. The incidence of infection (2.9\%) was low, without the use of prophylactic antibiotics. Skin necrosis (4.8\%) may be due to a number of factors. These would include a too tight dressing, use of adrenaline, delay in realising any haematoma (long lasting effect of bupicaine), skin degloving.

The number of patients lost to follow up (14.4\%) may be attributable to instruction given to the patients to return "should any concern arise". Preoperative information sheets are also used. Unfortunately, otoplasty is perceived as a minor procedure, and the patients often see no need to return for a visit if they had no particular problem to address.

In most significant and large studies the revision rate is between $0.03 \%-10 \%$ and the rate of complications $3 \%$ $15 \%$ with patient satisfaction rate maximum $85 \%$. Anterior scoring is the most commonly used technique in the UK for correction of prominent ears.

Tan [3] compared posterior suturing techniques with anterior scoring procedures in terms of the incidence and the severity of complications. The author found a significantly large number of patients treated by the posterior suture method of Mustardé required reoperation 24\% versus $10 \%$ with the anterior scoring technique. However, fewer postoperative haematoma and skin necrosis.

In a 10 year survey of his results with 264 ears, Mustarde' [7] lists several potential problems with otoplasty procedure that bears his name. A subsequent review of his results in 600 ears treated over 20 years revealed remarkably, only 10 ears became prominent again that they had to be reoperated.

Messner and Crysdale [8] review their experience with a combination technique of Mustardé and Furnas sutures in 31 patients followed for a minimum of 1 year (average 3.7 years). With regard to recurrence of the deformity, they report that at the time of evaluation one third of ears had returned to their original position, one third of ears remained in their immediately postoperative position, and one third of ears had final positions between the preand postoperative positions.

Calder and Naasan [9] reviewed their experience in 562 otoplasties by the anterior scoring technique. The most common complication was residual deformity, which occurred in $8 \%$ of patients. The aetiology of residual deformity was judged to be primarily a fault in the design of the procedure or in the execution of the technique. The authors believe most of these complications are avoidable.

This study confirms that this operation, like all operations, has a learning curve. The relationship between complication rate and grade of surgeon has previously been described [10]. It is however interesting to note that junior surgeons, if supervised, can achieve as good results as, if not better, than consultants. Although the number of procedures performed by Senior House Officer has significantly decreased in the recent years. Only 2 (1.92\%) operations were performed by SHO in our unit in the last 2 years, in contrast to previous studies in other units in the past $(26 \%$ - 28\%) $[9,10]$.

This project was started as basic clinical audit of process and outcome that can be used as a baseline of results against which future results can be compared. The complication rate in this series is similar to this of other reported studies. The overall satisfaction rate is good [8-14]. No technique is without complications or limitations.

Surgeons who treat this deformity must have a thorough understanding of the anatomy of the normal and prominent ear, be able to correctly and precisely analyze the deformity, and be able to establish and implement a surgical plan based on the available techniques. It is preferable that the surgeon is comfortable with multiple techniques. Following this audit, the protocol for the treatment of prominent ears has changed. These are:

1) Non steroidal anti inflammatories not given for $8 \mathrm{hrs}$ after the surgery.

2) Short acting local anaesthetic ( $0.5 \%$ lignocaine in 1:200,000 adrenaline) used in minimal amounts.

3) A posterior suturing technique (Mustardé and Furnas) with adipo-fascial flap [15].

4) Minimum dressing.

This protocol is to be reaudited.

\section{REFERENCES}

[1] P. A. Adamson and H. D. Strecker, "Otoplasty Techniques,” Facial Plastic Surgery, Vol. 11, No. 4, 1995, pp. 284-300. doi:10.1055/s-2008-1064545

[2] M. Spira, "Otoplasty: What I Do Now-A 30 Year Perspective," Plastic and Reconstructive Surgery, Vol. 104, No. 3, 1999, pp. 834-841.

doi:10.1097/00006534-199909030-00036

[3] K. H. Tan, "Long-Term Survey of Prominent Ears Surgery: A Comparison of Two Methods,” British Journal of Plastic Surgery, Vol. 39, No. 2, 1986, pp. 270-273. doi:10.1016/0007-1226(86)90100-1

[4] S. J. Stenstrom and J. Heftner, "The Stenstrom Otoplasty,” Clinics in Plastic Surgery, Vol. 5, No. 3, 1978, pp. 465-470.

[5] V. Chongchet, "A Method of Antihelix Reconstruction," 
British Journal of Plastic Surgery, Vol. 16, 1963, pp. 268-272. doi:10.1016/S0007-1226(63)80120-4

[6] A. J. McDowell, "Goals in Otoplasty for Protruding Ears,” Plastic and Reconstructive Surgery, Vol. 41, No. 1, 1968, pp. 17-27. doi:10.1097/00006534-196801000-00004

[7] J. C. Mustardé, “The Treatment of Prominent Ears by Buried Mattress Sutures-A Ten Years' Survey,” Plastic and Reconstructive Surgery, Vol. 39, No. 4, 1967, pp. 382-386. doi:10.1097/00006534-196704000-00008

[8] A. H. Messner and W. S. Crysdale, "Otoplasty: Clinical Protocol and Long-Term Results," Archives of Otolaryngology_Head and Neck Surgery, Vol. 122, No. 7, 1996, pp. 773-777.

doi:10.1001/archotol.1996.01890190069016

[9] J. C. Calder and A. Naasan, "Morbidity of Otoplasty: A Review of 562 Consecutive Cases," British Journal of Plastic Surgery, Vol. 47, No. 3, 1984, pp. 170-174. doi:10.1016/0007-1226(94)90049-3

[10] S. L. A. Jeffery, "Complications Following Correction of Prominent Ears: An Audit Review of 122 Cases,” British Journal of Plastic Surgery, Vol. 52, No. 7, 1999, pp. 558590.

[11] K. E. Graham and D. T. Gault, "Endoscopic Assisted Otoplasty: A Preliminary Report,” British Journal of Pla- stic Surgery, Vol. 50, No. 1, 1997, pp. 47-57. doi:10.1016/S0007-1226(97)91283-2

[12] N. W. Bulstrode, S. Huang and D. L. Martin, "Otoplasty by Percutaneous Anterior Scoring. Another Twist to the Story: A Long-Term Study of 114 Patients,” British Journal of Plastic Surgery, Vol. 56, No. 2, 2003, pp. 145-149. doi:10.1016/S0007-1226(03)00030-4

[13] L. Caouette-Laberge, N. Guay, M. D. Bortoluzzi and C. Belleville, "Otoplasty: Anterior Scoring Technique and Results in 500 Cases," Plastic and Reconstructive Surgery, Vol. 105, No. 2, 2000, pp. 504-515. doi:10.1097/00006534-200002000-00004

[14] P. Yugueros and J. Friedland, "Otoplasty: The Experience of 100 Consecutive Patients," Plastic and Reconstructive Surgery, Vol. 108, No. 4, 2001, pp. 1045-1051. doi:10.1097/00006534-200109150-00038

[15] N. Horloch, A. Misra and D. T. Gault, "The Postauricular Fascial Flap as an Adjunct to Mustardé and Furnas Type Otoplasty,” Plastic and Reconstructive Surgery, Vol. 108, No. 5, 2001, pp. 1487-1490. 